\title{
Emission of SN 1006 produced by accelerated cosmic rays
}

\author{
E. G. Berezhko ${ }^{1}$, L. T. Ksenofontov ${ }^{1}$, and H. J. Völk ${ }^{2}$ \\ 1 Institute of Cosmophysical Research and Aeronomy, 31 Lenin Av., 677891 Yakutsk, Russia \\ e-mail: berezhko@ikfia.ysn.ru e-mail: ksenofon@ikfia.ysn.ru \\ 2 Max Planck Institut für Kernphysik, Postfach 103980, 69029 Heidelberg, Germany
}

Received 8 April 2002 / Accepted 23 August 2002

\begin{abstract}
The nonlinear kinetic model of cosmic ray (CR) acceleration in supernova remnants (SNRs) is used to describe the properties of the remnant of SN 1006. It is shown, that the theory fits the existing data in a satisfactory way within a set of parameters which is consistent with the idea that SN 1006 is a typical source of Galactic CR nucleons, although not necessarily of CR electrons. The adjusted parameters are those that are not very well determined by present theory or not directly amenable to astronomical observations. The calculated expansion law and the radio-, X-ray and $\gamma$-ray emissions produced by the accelerated CRs in SN 1006 agree quite well with the observations. A rather large interior magnetic field $B_{\mathrm{d}} \approx 100 \mu \mathrm{G}$ is required to give a good fit for the radio and X-ray synchrotron emission. In the predicted TeV $\gamma$-ray flux from SN 1006, the $\pi^{0}$-decay $\gamma$-rays, generated by the nuclear CR component, dominate over the inverse Compton (IC) $\gamma$-rays, generated by the CR electrons in the cosmic microwave background. The predicted source morphology in high energy $\gamma$-rays roughly corresponds to that of the synchrotron emission. The predicted integral $\gamma$-ray flux $F_{\gamma} \propto \epsilon_{\gamma}^{-1}$ extends up to energies $\sim 100 \mathrm{TeV}$ if $\mathrm{CR}$ diffusion is as strong as the Bohm limit. Only if the interior magnetic field is much lower in the $\mathrm{SNR}, B_{\mathrm{d}} \approx 10 \mu \mathrm{G}$, then the observed $\gamma$-ray emission can be due to the accelerated electron component alone. In this case, not plausible physically in our view, the lowest permissible value of the electron to proton ratio is rather high, and the maximum individual energy and total energy content of accelerated nucleons so small, that SN 1006 can not be considered as a typical source of the nuclear Galactic CRs.
\end{abstract}

Key words. ISM: cosmic rays - acceleration of particles - stars: supernovae: individual: SN 1006 - radio continuum: ISM $\mathrm{X}$-rays: ISM - gamma rays: theory

\section{Introduction}

In the last years significant efforts have been made to obtain direct observational evidence whether the Galactic cosmic rays (CRs) - relativistic nucleons and electrons - are indeed generated in supernova remnants (SNRs). The expected $\pi^{0}$-decay $\gamma$-ray emission, produced in nearby SNRs by the accelerated protons in their collisions with thermal gas nuclei, is marginally high enough to be detectable by the present generation of imaging atmospheric Cherenkov telescopes (e.g. Drury et al. 1994; Naito \& Takahara 1994; Berezhko \& Völk 1997). Positive results of such observations would constitute a necessary condition for a dominant role of SNRs in the production of the Galactic CRs and of their energy spectrum up to the knee energy $\sim 10^{15} \mathrm{eV}$.

If SNRs accelerate nucleons at least to $100 \mathrm{TeV} / n$ as it is required for Galactic CRs (e.g Berezhko \& Ksenofontov 1999), then in smooth extension of the expected hadronically generated $\pi^{0}$-decay $\gamma$-ray peak at $67.5 \mathrm{MeV}$ (e.g. Stecker 1971; Dermer 1986) one expects the nucleonic $\gamma$-ray spectrum to have an almost single power-law form to about $1 \mathrm{TeV}$,

Send offprint requests to: $\mathrm{H}$. J. Völk,

e-mail: Heinrich.Voelk@mpi-hd.mpg.de before turning over above $10 \mathrm{TeV}$. At the same time the leptonic Inverse Compton (IC) $\gamma$-ray spectrum may be quenched at a lower cut-off energy due to electron synchrotron losses (as in our model). Then $\gamma$-ray observations within the range from $10 \mathrm{MeV}$ to $100 \mathrm{TeV}$ would be useful in discriminating between the nucleonic and leptonic components.

Recent observations of nonthermal X-rays and $\gamma$-rays indicate that at least CR electrons are accelerated in SNRs. SN 1006 is one of the SNRs for which there is evidence that electrons reach energies of about $100 \mathrm{TeV}$ (Koyama et al. 1995; Tanimori et al. 1998, 2001). It is also one of the three shell type SNRs in which $\mathrm{TeV} \gamma$-ray emission has been detected up to now. However, the interpretation of these data is not unique. Depending on the assumed values for the unknown physical parameters of SN 1006 (mainly the value of the magnetic field, the electron to proton ratio, and to some extent also the nucleon injection rate), the observed high-energy $\gamma$-ray emission can be predominantly either inverse Compton radiation due to CR electrons scattering on the microwave background radiation (as predicted by Mastichiadis 1996; Pohl 1996; Mastichiadis \& de Jager 1996; and Yoshida \& Yanagita 1997 from the X-ray synchrotron emission, and through the interpretation of the X-ray and $\gamma$-ray data by Aharonian 1999; 
Aharonian \& Atoyan 1999; Berezhko et al. 1999), or $\pi^{0}$-decay emission due to hadronic collisions of CRs with gas nuclei (Aharonian 1999; Aharonian \& Atoyan 1999; Berezhko et al. 1999; Berezhko et al. 2001).

The thermal and nonthermal X-ray emission has recently been rediscussed by Allen et al. (2001) who draw phenomenological conclusions on the $\gamma$-ray emission to be dominated by IC radiation as well as on the production and the characteristics of the nonthermal nucleonic particle component which they estimate to have a total energy of $10^{50} \mathrm{ergs}$.

In contrast, our starting point is the overall SNR dynamics and the acceleration theory of the nonthermal component. Thus we accept the X-ray results and the quantitative distinction between thermal X-ray emission and the nonthermal synchrotron components, including the constraints on the external density derived from them. We calculate the outer shock size, speed and compression ratio as well as the nonthermal quantities as solutions of the nonlinear, time-dependent kinetic equations for electrons and protons as functions of space, time and particle momentum, rather than phenomenologically assuming forms of the electron and proton momentum distribution functions.

In this way we also obtain full morphological information. We determine the unknown parameters like the upstream magnetic field strength and the electron to proton ratio, and constrain the nucleon injection rate which cannot yet be accuratetely calculated from known theory, by comparing with the observations. For this purpose we use the selfconsistent kinetic model of diffusive acceleration of CRs in SNRs (Berezhko et al. 1996; Berezhko \& Völk 1997) and investigate the $\gamma$-ray emission from SN 1006. A preliminary version of our results is given in Berezhko et al. (2001).

In contrast to a previous study (Berezhko et al. 1999) we restrict ourselves to the so-called Bohm limit for CR diffusion near the shock, assuming efficient and strong excitation of magnetohydrodynamic waves by the accelerating particles themselves. This is consistent with the large magnetic field strengths which we infer for this remnant.

Our considerations show that, together with a renormalization due to the lack of spherical symmetry of the nucleon injection, the existing SNR data are consistent with very efficient acceleration of CR nuclei at the SN shock wave which converts a significant fraction of the initial SNR energy content into CR energy as required for a typical source of the Galactic $\mathrm{CR}$ nuclei. The relative amount of energetic electrons is rather small, and may require additional sources to SNe type Ia like SN 1006. Therefore the observed $\gamma$-ray emission of SN 1006 can indeed be of hadronic origin, and we consider this as the physically most plausible solution from the point of view of acceleration theory.

Nevertheless, the existing observations do not strongly exclude a solution in which nuclear CRs play no important role and all nonthermal emissions are of leptonic origin. Therefore we analyze whether the existing data can also be fitted by an essentially different set of parameters. We demonstrate that there is an alternative possibility to fit the overall data, albeit with somewhat lower quality.

It implies a (physically not plausible) much lower injection rate and acceleration efficiency of protons, a rather large electron to proton ratio, and a lower magnetic field value compared with the case of efficient CR nucleon acceleration. If we would assume SN 1006 to be a typical representative, in this second case we could not consider the SNRs as the source population of the Galactic CRs due to the low proton acceleration efficiency. To discriminate empirically between these rather different scenarios from our results $\gamma$-ray measurements above $10 \mathrm{TeV}$ are required: in this very high energy range we expect a measurable $\gamma$-ray flux only in the case of efficient nucleonic CR production. We also confirm the earlier result (Aharonian 1999; Aharonian \& Atoyan 1999; Berezhko et al. 1999; Berezhko et al. 2001) that the IC $\gamma$-ray emission should cover the SNR almost uniformly, whereas the $\pi^{0}$-decay emission should be peaked behind the shock front and limited to two polar caps where the interstellar magnetic field is parallel to the shock normal.

\section{Model}

Since SN 1006 is a type Ia supernova (SN) we suggest that its evolution takes place in a uniform interstellar medium (ISM), not modified by a wind from the progenitor star. The general picture is then well-known.

A SN explosion ultimately ejects a shell of matter with total energy $E_{\mathrm{sn}}$ and mass $M_{\mathrm{ej}}$. During an initial period the shell material has a broad distribution in velocity $v$. The fastest part of these ejecta is described by a power law $\mathrm{d} M_{\mathrm{ej}} / \mathrm{d} v \propto v^{2-k}$ (e.g. Jones et al. 1981; Chevalier 1982). The interaction of the ejecta with the ISM creates a strong shock there which accelerates particles.

Our nonlinear model (Berezhko et al. 1996; Berezhko \& Völk 1997) is based on a fully time-dependent solution of the $\mathrm{CR}$ transport equation together with the gas dynamic equations in spherical symmetry. It yields at any given phase of the SNR evolution the complete spatial distribution of gas and accelerated CRs and their spectrum. This makes it possible to calculate any kind of emission, and its morphology, produced by the accelerated particles.

The CR diffusion coefficient is taken as the Bohm limit

$\kappa(p)=\kappa(m c)(p / m c)$,

where approximately $\kappa(m c)=m c^{2} /(3 e B), e$ and $m$ are the particle charge and mass, $p$ denotes the particle momentum, $B$ is the magnetic field strength, and $c$ is the speed of light. With this diffusion coefficient we assume that the production of scattering waves is so strong that limitations due to wave refraction (Malkov et al. 1995) do not come in to influence the solution.

The number of suprathermal protons injected into the acceleration process is described by a dimensionless injection parameter $\eta$ which is a fixed fraction of the ISM particles entering the shock front. For simplicity it is assumed that the injected particles have a velocity four times higher than the postshock sound speed. Unfortunately there is no complete selfconsistent theory of a collisionless shock transition which can predict the value of the injection rate and its dependence on the shock parameters. For the case of a purely parallel plane shock hybrid simulations predict a quite high ion injection (e.g. Scholer et al. 1992; Bennet \& Ellison 1995) which corresponds to the value 
$\eta \sim 10^{-2}$ of our injection parameter. Such a high injection is consistent with analytical models (Malkov \& Völk 1995, 1996; Malkov 1998) and confirmed by measurements near the Earth's bow shock (Trattner \& Scholer 1994). We note however that in our spherically symmetric model these results can only be used with some important modification. In reality we must consider the evolution of the large scale SN shock which expands into the ISM with its magnetic field. In the case of SN 1006, at the current evolutionary phase, the shock has a size of several parsecs. On such a scale the unshocked interstellar magnetic field can be considered as uniform since its random component is characterized by a much larger main scale of about $100 \mathrm{pc}$. Then our spherical shock is quasi-parallel in the polar regions and quasi-perpendicular in the equatorial region. This magnetic field essentially suppresses the leakage of suprathermal particles from the downstream region back upstream when the shock is more and more oblique (Ellison et al. 1995; Malkov \& Völk 1995). Applied to the spherical shock in the uniform external magnetic field it would mean that only small regions near the poles, covering less than $10 \%$ of the shock surface, allow a sufficiently high injection that ultimately leads to the transformation of an essential part (more than a few percent) of the shock energy into CR energy, whereas the main part of the shock is an inefficient CR accelerator. If one takes into account the Alfvén wave excitation due to CR streaming (which becomes efficient already at a very low injection rate $\eta \sim 10^{-7}$ ) the local injection rate has to be averaged over the fluctuating magnetic field directions and is lower than for the purely parallel case by a factor of hundred. Therefore we adopt here the value $\eta \sim 10^{-4}$ for the injection parameter (Völk et al. 2002). The detailed choice $\eta=2 \times 10^{-4}$ in the next section is made in order to achieve a nonlinear shock modification that is consistent with the observed radio spectral index.

According to our above estimate a substantial part of the shock still efficiently injects and accelerates CRs. This fraction is effectively increased relative to the above percentage due to broadening of the injection region by the strong wave field as well as by CR diffusion perpendicular to the mean magnetic field. In addition, the overall conservation equations ensure an approximately spherical character of the overall dynamics. Therefore, we assume the spherically symmetric approach for the nonlinear particle acceleration process to be approximately valid in those shock regions where injection is efficient. To take the effective injection fraction $f_{\text {re }}<1$ into account, we then need to introduce a renormalization factor for the CR acceleration efficiency and for all the effects which it produces in the SNR. A rough estimate performed for the simple case of spherical shock expended in the uniform outer magnetic field (Völk et al. 2002) gives $f_{\text {re }}=0.15$ to 0.25 .

Note that such a picture is consistent with the observed structure of SN 1006: the intense radio and X-ray emissions come from two bright rims with radially oriented magnetic field (Reynolds \& Gilmore 1993). They may be the polar regions of a quasispherical shock in an outer magnetic field. The $\gamma$-ray observations may indicate a similar asymmetry (Tanimori et al. 1998) which would be well explained by a corresponding concentration of the accelerated nucleons towards the polar regions.
We assume that electrons are also injected into the acceleration process, still at nonrelativistic energies below $m_{\mathrm{e}} c^{2}$. Since the electron injection mechanism is not very well known (e.g. Malkov \& Drury 2001) for simplicity we consider their acceleration starting from the same momentum as that of protons. At relativistic energies (for protons) these electrons have exactly the same dynamics as the protons. Therefore, neglecting synchrotron losses, their distribution function at any given time has the form

$f_{\mathrm{e}}(p)=K_{\mathrm{ep}} f(p)$

with some factor $K_{\text {ep }}$ which is about $10^{-2}$ for the average CRs in the Galaxy. We take it here as a parameter.

The electron distribution function $f_{\mathrm{e}}(p)$ deviates only at sufficiently large momenta from this relation due to synchrotron losses, which are taken into account by supplementing the ordinary diffusive transport equation with a loss term:

$$
\frac{\partial f_{\mathrm{e}}}{\partial t}=\nabla \kappa \nabla f_{\mathrm{e}}-\boldsymbol{w} \nabla f_{\mathrm{e}}+\frac{\nabla \boldsymbol{w}}{3} p \frac{\partial f_{\mathrm{e}}}{\partial p}-\frac{1}{p^{2}} \frac{\partial}{\partial p}\left(\frac{p^{3}}{\tau_{1}} f_{\mathrm{e}}\right),
$$

where the first three terms in the right hand side of this equation describe diffusion, convection due to the mass velocity $w$ of the gas and adiabatic effects, respectively. The synchrotron loss time in the third term is determined by the expression (e.g. Berezinskii et al. 1990)

$\tau_{1}=\left(\frac{4 r_{0}^{2} B^{2} p}{9 m_{\mathrm{e}}^{2} c^{2}}\right)^{-1}$

where $m_{\mathrm{e}}$ is the electron mass and $r_{0}$ the classical electron radius.

The solution of the dynamic equations at each instant of time yields the CR spectrum and the spatial distributions of CRs and gas. This allows us to calculate the expected flux $F_{\gamma}^{\pi}\left(\epsilon_{\gamma}\right)$ of $\gamma$-rays from $\pi^{0}$-decay due to hadronic (p-p) collisions of CRs with the gas nuclei. Following the work of Dermer (1986) and its later improvement by Naito \& Takahara (1994) we use here the isobar model at the protons kinetic energies $\epsilon_{k}<3 \mathrm{GeV}$ and the scaling model at $\epsilon_{k}>7 \mathrm{GeV}$ with a linear connection between 3 and $7 \mathrm{GeV}$. This model agrees very well with the simpler approach, introduced by Drury et al. (1994) (see also Berezhko \& Völk 1997, 2000; Berezhko et al. 1999) at high energies $\epsilon_{\gamma}>0.1 \mathrm{GeV}$, except in the cutoff region, where the scaling model yields an essentially smoother turnover of the $\gamma$-ray spectrum at somewhat lower energies.

The choice of $K_{\mathrm{ep}}$ allows us to determine in addition the electron distribution function and to calculate the associated emission. The expected synchrotron flux at distance $d$ from the SNR is given by the expression (e.g. Berezinskii et al. 1990)

$S_{v}=\frac{3 \times 10^{-21}}{d^{2}} \int_{0}^{\infty} \mathrm{d} r r^{2} B_{\perp} \int_{0}^{\infty} \mathrm{d} p p^{2} f_{\mathrm{e}}(r, p) F\left(\frac{v}{v_{\mathrm{c}}}\right)$

in $\mathrm{erg} /\left(\mathrm{cm}^{2} \mathrm{~s}\right)$, where

$F(x)=x \int_{x}^{\infty} K_{5 / 3}\left(x^{\prime}\right) \mathrm{d} x^{\prime}$

$K_{\mu}(x)$ is the modified Bessel function, $v_{\mathrm{c}}=3 e B_{\perp} p^{2} /\left[4 \pi\left(m_{\mathrm{e}} c\right)^{3}\right]$ and $B_{\perp}$ is the magnetic field component perpendicular to the line of sight. 
Since in the shock region where particle injection and acceleration is efficient the upstream magnetic field is assumed to be almost completely randomized due to intense Alfvén wave generation. For such a strongly turbulent upstream field, consistent with Bohm diffusion, the postshock field strength $B_{2}$ is given by by $B_{2}=\sigma B_{0}$, where $\sigma_{B}^{2}=1 / 3+(2 / 3) \sigma^{2}$. We shall approximately equate $\sigma_{B}$ with the gas compression ratio $\sigma$ at the shock that We suggest also that in such a young SNR like SN 1006 the downstream magnetic field $B_{\mathrm{d}}$ in a relatively thin region between the shock and the ejecta is approximately uniform and we shall take $B_{\mathrm{d}}=B\left(r<R_{\mathrm{S}}\right)=B_{2}$.

The relativistic electrons produce $\gamma$-ray emission due to IC scattering of background photons. It is not difficult to show that due to the hard spectrum of accelerated electrons only the $2.7 \mathrm{~K}$ cosmic microwave background (CMB) is important in the case considered. The expected differential flux of IC $\gamma$-rays as a function of their energy $\epsilon_{\gamma}$ can be represented in the form:

$$
\begin{aligned}
\frac{\mathrm{d} F_{\gamma}^{\mathrm{IC}}}{\mathrm{d} \epsilon_{\gamma}}= & \frac{4 \pi c}{d^{2}} \int_{0}^{\infty} \mathrm{d} r r^{2} \int_{0}^{\infty} \mathrm{d} \epsilon n_{\mathrm{ph}}(\epsilon) \\
& \times \int_{p_{\min }}^{\infty} \mathrm{d} p p^{2} \sigma\left(\epsilon_{\mathrm{e}}, \epsilon_{\gamma}, \epsilon\right) f_{\mathrm{e}}(r, p)
\end{aligned}
$$

in photons $/\left(\mathrm{cm}^{2} \mathrm{~s} \mathrm{erg}\right)$, where (Blumenthal \& Gould 1970)

$$
\begin{aligned}
\sigma\left(\epsilon_{\mathrm{e}}, \epsilon_{\gamma}, \epsilon\right) & =\frac{3 \sigma_{\mathrm{T}}\left(m_{\mathrm{e}} c^{2}\right)^{2}}{4 \epsilon \epsilon_{\mathrm{e}}^{2}} \\
\times & {\left[2 q \ln q+(1+2 q)(1-q)+0.5 \frac{(\Gamma q)^{2}(1-q)}{1+\Gamma q}\right] }
\end{aligned}
$$

is the differential cross section for the up-scattering of a photon with incident energy $\epsilon$ to energy $\epsilon_{\gamma}$ by the elastic collision with an electron of energy $\epsilon_{\mathrm{e}}$,

$n_{\mathrm{ph}}=\frac{1}{\pi^{2}(\hbar c)^{3}} \frac{\epsilon^{2}}{\exp \left(\epsilon / k_{\mathrm{B}} T\right)-1}$

is the blackbody spectrum of the $\mathrm{CMB}, \hbar$ and $k_{B}$ are Planck and Boltzmann constant respectively, $T=2.7 \mathrm{~K}, \sigma_{\mathrm{T}}=6.65 \times$ $10^{-25} \mathrm{~cm}^{2}$ is the Thomson cross-section, $q=\epsilon_{\gamma} /\left[\Gamma\left(\epsilon_{\mathrm{e}}-\epsilon_{\gamma}\right)\right], \Gamma=$ $4 \epsilon \epsilon_{\mathrm{e}} /\left(m_{\mathrm{e}} c^{2}\right)^{2}$, and $p_{\min }$ is the minimal momentum of electron, whose energy $\epsilon_{\mathrm{e}}$ is determined by the condition $q=1$.

Energetic electrons produce also nonthermal bremstrahlung (NB) emission, interacting with both ambient electrons and protons. We use standard formulae for the cross section to calculate the NB $\gamma$-ray flux $F_{\gamma}^{\mathrm{NB}}\left(\epsilon_{\gamma}\right)$ (e.g. Baring et al. 1999). As it was already demonstrated by these last authors, in young SNRs the primary energetic electron number density due to electrons directly accelerated from the suprathermal postshock distribution by far exceeds that of secondaries which were produced via the decay of charged pions created in $\mathrm{p}-\mathrm{p}$ collisions. Hence the contribution of secondaries to the NB, IC, and synchrotron emission can be neglected.

\section{Results and discussion}

Since SN 1006 is a type Ia SN we use typical SN Ia parameters in our calculations: ejected mass $M_{\mathrm{ej}}=1.4 M_{\odot}, k=7$, and a uniform ambient ISM.
The ISM density is the most relevant parameter, especially for the $\gamma$-ray production (e.g. Berezhko et al. 1999). Here we use the most appropriate value of the ambient number density of ISM hydrogen $N_{\mathrm{H}}=0.3 \mathrm{~cm}^{-3}$ as well as the distance $d=1.8 \mathrm{kpc}$, consistent with X-ray and optical imagery of SN 1006 (Winkler \& Long 1997, see also Allen et al. 2001 and references therein).

The value $T_{0}=10^{4} \mathrm{~K}$ is used for the ISM temperature. Note that SNR and CR dynamics are not sensitive to $T_{0}$, because the shock structure is mainly determined by the Alfvénic Mach number.

The other important parameter is the ISM magnetic field $B_{0}$. Unfortunately there is no direct way to determine its value from the observations. Since it influences the synchrotron spectrum in an essential way, we use an upstream magnetic field value $B_{0}=20 \mu \mathrm{G}$. It is required to provide the observed shape of the synchrotron spectrum in the radio and the X-ray bands.

The gas dynamics problem is characterized by the following length, time, and velocity scales:

$R_{0}=\left(3 M_{\mathrm{ej}} / 4 \pi \rho_{0}\right)^{1 / 3}, t_{0}=R_{0} / V_{0}, V_{0}=\sqrt{2 E_{\mathrm{sn}} / M_{\mathrm{ej}}}$,

which are the sweep-up radius, sweep-up time and mean ejecta speed respectively. Here $\rho_{0}=1.4 m_{\mathrm{p}} N_{\mathrm{H}}$ is the ISM mass density; $m_{\mathrm{p}}$ is the proton mass.

The shock expansion law during the free expansion phase $\left(t<t_{0}\right)$ is then

$R_{\mathrm{s}} \propto E_{\mathrm{sn}}^{(k-3) / 2 k} \rho_{0}^{-1 / k} t^{(k-3) /(k-2)}$

(Chevalier 1982) which for $k=7$ gives

$R_{\mathrm{S}} \propto\left(E_{\mathrm{sn}}^{2} / \rho_{0}\right)^{1 / 7} t^{4 / 5}$.

In the adiabatic phase $\left(t \gtrsim t_{0}\right)$ we have

$R_{\mathrm{S}} \propto\left(E_{\mathrm{sn}} / \rho_{0}\right)^{1 / 5} t^{2 / 5}$.

The observed expansion law of SN 1006 (Moffett et al. 1993) is $R_{\mathrm{S}} \propto t^{\mu}$ with $\mu=0.48 \pm 0.13$. We conclude that within the observational errors SN 1006 should already be in the adiabatic phase.

The calculations together with the experimental data are shown in Figs. 1-5. For the assumed SN distance and ISM density an explosion energy $E_{\mathrm{sn}}=3 \times 10^{51}$ erg fits the observed SNR size $R_{\mathrm{S}}$ and its expansion rate $V_{\mathrm{S}}$ (Moffet et al. 1993). The shown uncertainties of $R_{\mathrm{S}}$ and $V_{\mathrm{s}}$ are calculated as the uncertainties of the angular size and expansion rate times the distance $d=1.8 \mathrm{kpc}$.

According to Fig. 1a SN 1006 is indeed already in the adiabatic phase. The assumed injection rate $\eta=2 \times 10^{-4}$ leads to a significant modification of the shock which at the current epoch, $t=995 \mathrm{yr}$, has a total compression ratio $\sigma=6.3$ and a subshock compression ratio $\sigma_{\mathrm{s}}=3.6$ (Fig. 1b).

The different SNR energy components, the ejecta energy $E_{\mathrm{ej}}$, the gas kinetic $\left(E_{\mathrm{gk}}\right)$, and thermal $\left(E_{\mathrm{gt}}\right)$ energies, and the CR energy $E_{\mathrm{c}}$, are presented in Fig. $1 \mathrm{c}$ as functions of time. The acceleration process is characterized by a high efficiency in spherical symmetry: at the current time $t / t_{0}=4.68$ 


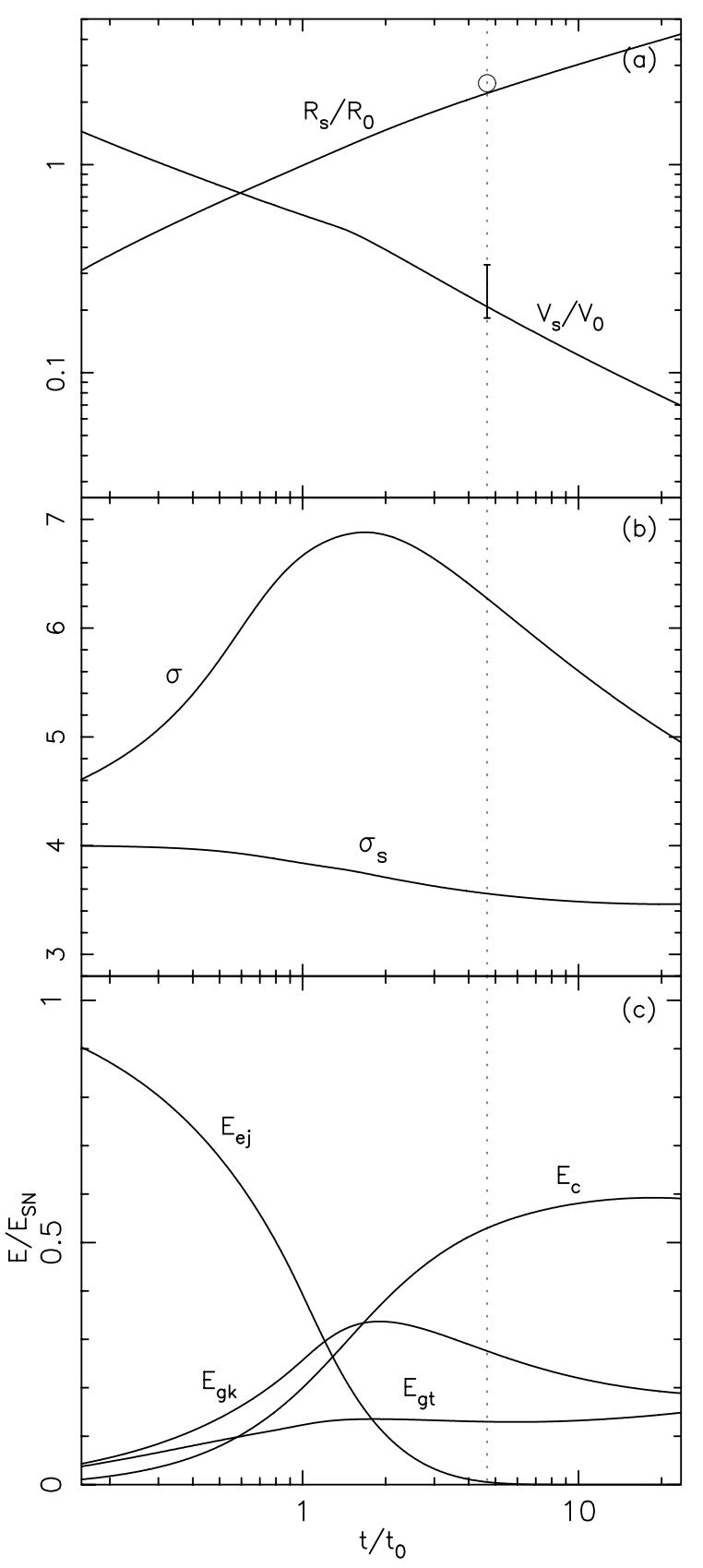

Fig. 1. a) Shock radius $R_{\mathrm{s}}$ and shock speed $V_{\mathrm{s}}$; b) total shock $(\sigma)$ and subshock $\left(\sigma_{\mathrm{s}}\right)$ compression ratios; c) ejecta $\left(E_{\mathrm{ej}}\right), \mathrm{CR}\left(E_{\mathrm{c}}\right)$, gas thermal $\left(E_{\mathrm{gt}}\right)$, and gas kinetic $\left(E_{\mathrm{gk}}\right)$ energies as a function of time. Scale values are $R_{0}=3.2 \mathrm{pc}, V_{0}=14675 \mathrm{~km} \mathrm{~s}^{-1}, t_{0}=212$ years. The dotted vertical line marks the current epoch. The observed size and speed of the shock (Moffett et al. 1993), are shown as well.

about $53 \%$ of the explosion energy has been already transferred to CRs, and the CR energy content $E_{\mathrm{c}}$ continues to increase to a maximum of about $60 \%$ in the later Sedov phase (Fig. 1c), when particles start to leave the source. As usually predicted by the model, the CR acceleration efficiency is significantly higher than required for the average replenishment of the Galactic CRs by SNRs, corresponding to $E_{\mathrm{c}} \approx 0.1 E_{\mathrm{sn}}$. As discussed before, this discrepancy can be attributed to the physical conditions at the shock surface which influence the injection efficiency. The magnetic field geometry is the most

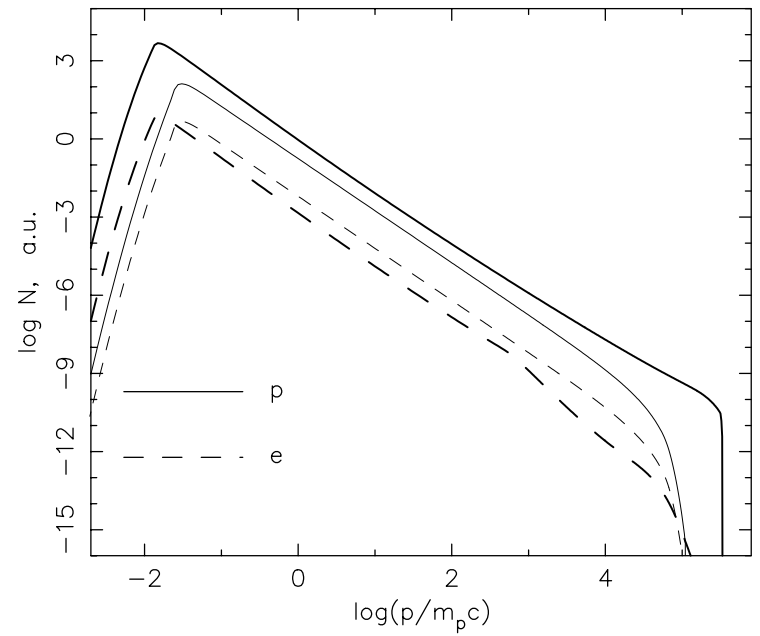

Fig. 2. The overall (= spatially integrated) CR spectrum as function of momentum. Solid and dashed lines correspond to protons and electrons, respectively. Thick and thin lines correspond to efficient and inefficient proton acceleration, respectively.

important factor: at the quasiperpendicular portion of the shock ion injection (and subsequent acceleration) is presumably depressed compared with the quasiparallel portion. Therefore the number of nuclear CRs, calculated within the sphericallysymmetrical approximation, should be renormalized by this depression factor (see Völk et al. 2002 for details). Assuming SN 1006 to be an average Galactic CR source, the renormalizing factor should be $f_{\text {re }}=0.2$. It means that according to our calculation the actual energy of accelerated protons in SN 1006 at the present epoch is

$E_{\mathrm{c}}=0.53 f_{\mathrm{re}} E_{\mathrm{sn}}=3.2 \times 10^{50} \mathrm{erg}$.

Note that such a factor is consistent with observations which show that efficient CR acceleration takes place at about $25 \%$ of the shock surface (e.g. Allen et al. 2001).

The volume-integrated (or overall) CR spectrum

$N(p, t)=16 \pi^{2} p^{2} \int_{0}^{\infty} \mathrm{d} r r^{2} f(r, p, t)$

has, for the case of protons, almost a pure power-law form $N \propto p^{-\gamma}$ over a wide momentum range from $10^{-2} m_{\mathrm{p}} c$ up to the cutoff momentum $p_{\max }=\epsilon_{\max } / c$, where $\epsilon_{\max } \approx 3 \times 10^{14} \mathrm{eV}$ is the maximum CR energy (Fig. 2). The limitation of this numerically determined value $p_{\max }$ can be understood mainly in terms of the influence of geometrical factors which are the finite size and speed of the shock, its deceleration and the adiabatic cooling effect in the downstream region (Berezhko 1996). Due to the shock modification the power-law index slowly varies from $\gamma=2.1$ at $p \lesssim m_{\mathrm{p}} c$ to $\gamma=1.8$ at $p \gtrsim 10^{3} m_{\mathrm{p}} c$.

The shape of the overall electron spectrum $N_{\mathrm{e}}(p)$ deviates from that of the proton spectrum $N(p)$ at high momenta $p>p_{1} \approx 10^{3} m_{\mathrm{p}} c$, due to the synchrotron losses in the downstream region with magnetic field $B_{\mathrm{d}} \approx 120 \mu \mathrm{G}$ which is assumed uniform in this region $\left(B_{\mathrm{d}} \approx B_{2}=\sigma B_{0}\right)$. According to 
expression (4) the synchrotron losses should become important for electron momenta greater than

$$
\frac{p_{1}}{m_{\mathrm{p}} c} \approx 1.3\left(\frac{10^{8} \mathrm{yr}}{t}\right)\left(\frac{10 \mu \mathrm{G}}{B_{\mathrm{d}}}\right)^{2} .
$$

Substituting the SN age $t=10^{3} \mathrm{yr}$ into this expression, we have $p_{1} \approx 600 m_{\mathrm{p}} c$, in reasonable agreement with the numerical results (Fig. 2).

In detail the momentum dependence of the spatially integrated electron spectrum $N_{\mathrm{e}}$ from Eq. (13) is somewhat more complicated at high energies. This can be understood as follows:

The shock constantly produces the electron spectrum $f_{\mathrm{e}} \propto$ $p^{-q}$ with $q \approx 4$ up to the maximum momentum $p_{\max }^{\mathrm{e}}$ which is much larger than $p_{1}$. This spectrum is not modified by synchrotron losses within the downstream region of thickness

$l=\tau_{1} u_{2}$,

where $u_{2}=V_{\mathrm{s}} / \sigma$ is the gas speed with respect to the shock. At low momenta $p<p_{1}$ the loss time $\tau_{1}$ exceeds the age of the system. Formally this means that the size of the lossfree region is greater than the thickness $\Delta r \approx R_{\mathrm{S}} /(3 \sigma)$ of the swept up shell. At large momenta $p>p_{1}$ we have $l<\Delta r$. Within the downstream region $r<R_{\mathrm{S}}-l$ the electron spectrum therefore becomes essentially modified $\left(f_{\mathrm{e}} \propto p^{-5}\right)$ by the influence of the synchrotron losses. At a given radius $r<R_{\mathrm{s}}$ accelerated electrons have an age of $\Delta t \approx\left(R_{\mathrm{s}}-r\right) / u_{2}$ and therefore, cf. Eq. (14), their spectrum has a break at $p_{1}(r)=p_{1} \times(t / \Delta t)$. According to its definition (13) the spatially integrated electron spectrum $N_{\mathrm{e}}(p)$ is a mixture of electron spectra with different $p_{1}(r)$. Qualitatively one would expect that within the momentum range $p_{1}$ to $p_{\max }^{\mathrm{e}}$, the overall electron spectrum is close to the form $N_{\mathrm{e}} \propto p^{-3}$, somewhat hardening towards $p_{\max }^{\mathrm{e}}$. This is in agreement with the calculation.

The maximum electron momentum $p_{\max }^{\mathrm{e}}$ can be estimated by equating the synchrotron loss time (4) and the acceleration time

$\tau_{\mathrm{a}}=\frac{3}{\Delta u}\left(\frac{\kappa_{1}}{u_{1}}+\frac{\kappa_{2}}{u_{2}}\right)$,

where $u_{1}=V_{\mathrm{s}}$ and $u_{2}=u_{1} / \sigma$ are the upstream and downstream gas velocities relative to the shock front, and $\Delta u=$ $u_{1}-u_{2}$. During the acceleration time $\tau_{\mathrm{a}}$ the electrons spent the time $3 \kappa_{1} /\left(u_{1} \Delta u\right)$ in the upstream magnetic field $B_{0}$, and $3 \kappa_{2} /\left(u_{2} \Delta u\right)$ in the downstream magnetic field $B_{2}=\sigma_{\mathrm{B}} B_{0}$ (in the case considered $\sigma_{\mathrm{B}}=\sigma$ ). Therefore, in the general case the maximum electron momentum $p_{\max }^{\mathrm{e}}$ is a solution of the equation

$\tau_{\mathrm{a}}(p)^{-1}=\left[\tau_{1}^{-1}\left(B_{0}, p\right)+\tau_{1}^{-1}\left(B_{2}, p\right) \sigma / \sigma_{\mathrm{B}}\right] /\left(1+\sigma / \sigma_{\mathrm{B}}\right)$,

which can be written in the form

$$
\begin{aligned}
\frac{p_{\max }^{\mathrm{e}}}{m_{\mathrm{p}} c}= & 6.7 \times 10^{4}\left(\frac{V_{\mathrm{s}}}{10^{3} \mathrm{~km} \mathrm{~s}^{-1}}\right) \\
& \times \sqrt{\frac{(\sigma-1)}{\sigma\left(1+\sigma_{\mathrm{B}} \sigma\right)}\left(\frac{10 \mu \mathrm{G}}{B_{0}}\right)} .
\end{aligned}
$$

The main part of the electrons with the highest energies $\epsilon_{\mathrm{e}} \gtrsim 10 \mathrm{TeV}$ is produced at the end of the free expansion phase. At this stage $V_{\mathrm{s}} \sim V_{0}$ which leads to a maximum electron momentum $p_{\max }^{\mathrm{e}} \approx 4 \times 10^{4} m_{\mathrm{p}} c$ in agreement with the numerical results (Fig. 2).

The parameters $K_{\mathrm{ep}}=3 \times 10^{-4}$ and $B_{\perp} \approx 0.3 B_{\mathrm{d}} \approx 36 \mu \mathrm{G}$ provide good agreement between the calculated and the measured synchrotron emission in the radio- and X-ray ranges (Fig. 3). The steepening of the electron spectrum at high energies due to synchrotron losses naturally yields a fit to the X-ray data with their soft spectrum. Note that two kinds of X-ray data are presented in Fig. 3. The Hamilton et al. (1986) data represent the total (i.e. thermal plus nonthermal) X-ray emission in a relatively wide frequency range, where the nonthermal synchrotron emission contribution dominates at frequencies $v \gtrsim 10^{18} \mathrm{~Hz}$. At these highest frequencies the Allen et al. (1999) RXTE nonthermal data are also presented (in red color), lying slightly below the Hamilton et al. data points. Clearly our nonthermal model spectra must lie below the Hamilton et al. spectral points at $v \lesssim 10^{18} \mathrm{~Hz}$. Such a smooth spectral behavior is achieved in a $20 \mu \mathrm{G}$ upstream field.

Since the total number of accelerated protons has to be reduced by the factor $f_{\mathrm{re}}=0.2$, the same number of accelerated electrons then corresponds to a renormalized parameter $K_{\text {ep }}=1.5 \times 10^{-3}$. Then the total energy of accelerated electrons in SN 1006 is $E_{\mathrm{c}}^{\mathrm{e}}=5 \times 10^{47} \mathrm{erg}$.

The ratio of accelerated electrons to protons thus comes out lower by a factor of about 6 than the canonical ratio of 0.01 , generally deduced from observations of the Galactic CRs. This is an interesting consequence of our model: if SN 1006 is typical for the nuclear Galactic CR source population, then other sources like young Pulsars or Pulsar Nebulae must also significantly contribute to the Galactic CR electron population (e.g. Aharonian et al. 1995; Pohl \& Esposito 1998). An additional fraction might come from secondary interstellar electrons/positrons due to the decay of charged pions that are produced in inelastic collisions of CR nuclei with nuclei of the interstellar gas. Since the majority of Galactic Supernovae is not of the type Ia to which SN 1006 belongs, but rather occurs as a consequence of core collapse events, there is the alternative possibility that core collapse Supernovae produce a higher electron to proton ratio. Such an increase might have to do with the different circumstellar magnetic field structure of massive stars, but this is indeed only a possibility for which we have no proof at present.

The radio data are fitted by a power law spectrum $S_{v} \propto v^{-\alpha}$, whose index $\alpha=0.57 \pm 0.06$ (Allen et al. 2001) is noticeably larger than 0.5 , corresponding to the electron spectrum $N_{\mathrm{e}} \propto p^{-2}$ produced by an unmodified shock with compression ratio $\sigma=4$. In our case the shock is essentially modified by the backreaction of the accelerated protons (see Fig. 1b): its compression ratio $\sigma=6.3$. At the same time low energy electrons with momenta $p \lesssim 10 m_{\mathrm{p}} c\left(\epsilon_{\mathrm{e}} \lesssim 10 \mathrm{GeV}\right)$, which produce synchrotron emission at $v \lesssim 10 \mathrm{GHz}$, are accelerated at the $s u b$ shock which has the compression ratio $\sigma_{\mathrm{s}}=3.6$. Therefore these electrons have a steeper spectrum $N_{\mathrm{e}} \propto p^{-2.1}$ that leads to the expected radio spectrum $S_{v} \propto v^{-0.54}$, fitting the experimental data very well (Fig. 3). The fact that the observed 


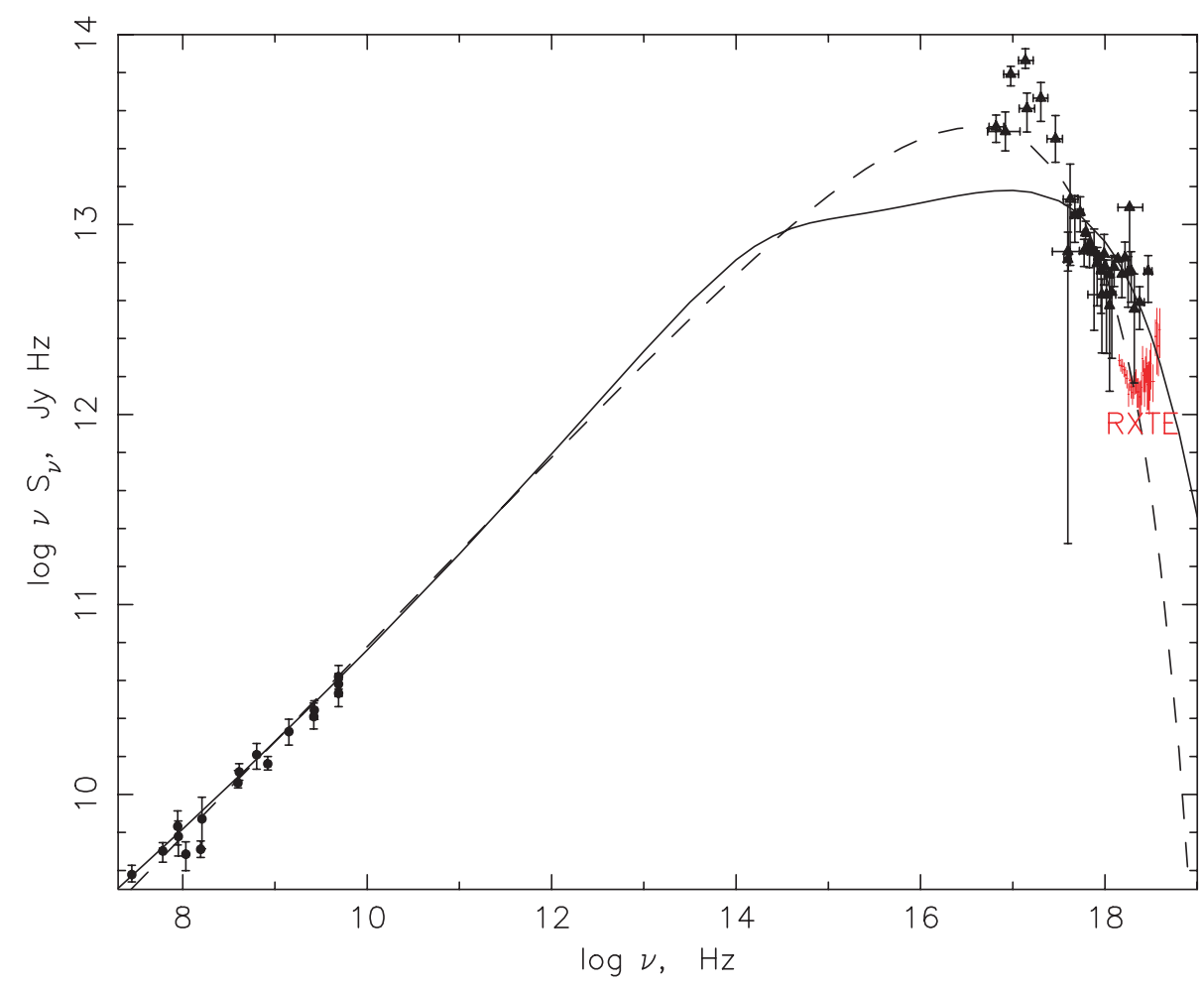

Fig. 3. Synchrotron emission flux as a function of frequency for the same two cases as in Fig. 2. Solid and dashed lines correspond to efficient and inefficient proton acceleration, respectively. The observed X-ray (in black color: Hamilton et al. 1986; in red color: Allen et al. 1999) and radio emissions (in black color: Reynolds 1996) are shown. value of the radio power law index $\alpha$ exceeds the value 0.5 can be considered as an indication that the shock is essentially modified. A relatively high upstream magnetic field strength $B_{0}=20 \mu \mathrm{G}$, compared with typical ISM values $B_{0}=5 \mu \mathrm{G}$, and a corresponding downstream value $B_{\perp}=36 \mu \mathrm{G}$ are required to have radio emitting electron energies in the steep part of their spectrum $\epsilon_{\mathrm{e}} \lesssim 1 \mathrm{GeV}$ on the one hand, and to give a smooth rollover of the synchrotron spectrum $S_{v}(v)$ at frequencies $v=10^{15}$ to $10^{18} \mathrm{~Hz}$ on the other. According to model calculations by Lucek \& Bell (2000), the existing ISM field can indeed be significantly amplified near a strong shock by CR streaming.

Such a strongly nonlinear behavior has three consequences in that (i) it leads to the high magnetic field strength, required to understand the measured nonthermal synchrotron spectrum, (ii) it is consistent with our assumption of Bohm diffusion, and (iii) it suggests a value $\eta \sim 10^{-4}$ for the injection parameter that we use (see also Sect. 2).

The calculated integral IC and $\pi^{0}$-decay $\gamma$-ray energy fluxes are presented in Figs. 4 and 5 together with the available experimental data.

In Fig. 4 we present also the integral NB $\gamma$-ray fluxes; they play no important role for SN 1006. Note that the number of accelerated protons, which produce $\pi^{0}$-decay $\gamma$-rays, has been reduced by the factor $f_{\text {re }}=0.2$.

According to the calculation, the hadronic $\gamma$-ray production exceeds the electron contribution by a factor of about 7 at energies $\epsilon_{\gamma} \lesssim 1 \mathrm{TeV}$, and dominates at $\epsilon_{\gamma}>10 \mathrm{TeV}$ (Fig. 4). The calculation is in good agreement with the TeV-measurements reported by the CANGAROO collaboration (Tanimori et al. 1998), and it does not contradict the EGRET upper limit

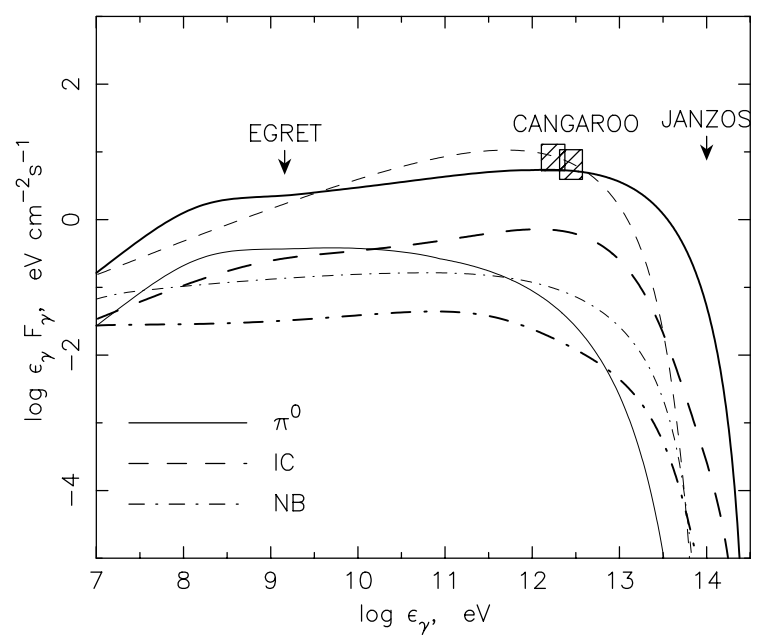

Fig. 4. IC (dashed lines), $\pi^{0}$-decay (solid lines) and NB (dash-dotted lines) integral $\gamma$-ray energy fluxes as a function of $\gamma$-ray energy for the same cases as in Fig. 2. High energy $\gamma$-ray data (Tanimori et al. 1998), the EGRET upper limit as given by Mastichiadis \& de Jager (1996), and the JANZOS upper limit (Allen et al. 1995) are shown.

$F_{\gamma}^{\mathrm{E}}=8 \times 10^{-9} \mathrm{~cm}^{-2} \mathrm{~s}^{-1}$ at $\epsilon_{\gamma}=1.4 \mathrm{GeV}$ (cf. Mastichiadis $\&$ de Jager 1996). This is also confirmed by Fig. 5, where we compare our calculations with the revised CANGAROO data (Tanimori et al. 2001) plus an extended set of EGRET upper limits (Naito et al. 1999).

The differential $\pi^{0}$-decay $\gamma$-ray flux $\mathrm{d} F_{\gamma}^{\pi} / \mathrm{d} \epsilon_{\gamma}$ has the expected peak at photon energy $m_{\pi} / 2=67.5 \mathrm{MeV}$ (see also Naito $\&$ Takahara 1994). This feature may be used for the identification of a hadronic contribution to the observed $\gamma$-ray flux at the particle energies $<10 \mathrm{GeV}$ mainly producing it. However, two considerations weaken this possibility. First of all, for 


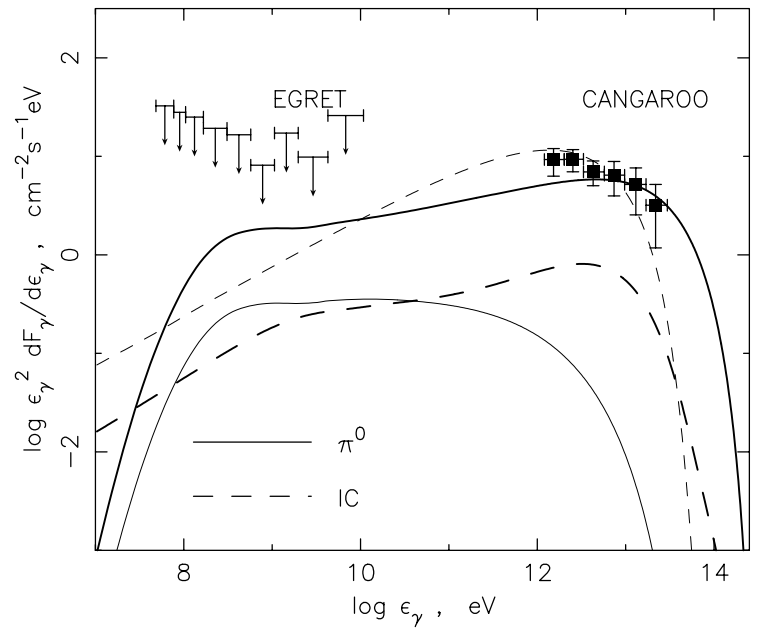

Fig. 5. Differential $\pi^{0}$-decay (solid lines) and IC (dashed lines) $\gamma$-ray energy fluxes as a function of $\gamma$-ray energy for the same cases as in Fig. 2. The recent differential high energy $\gamma$-ray energy flux data (Tanimori et al. 2001) and EGRET upper limits (Naito et al. 1999) are also shown.

reasons of instrumental sensitivity, we expect only nearby SNRs to be detectable in the foreseeable future. Such objects have diameters approaching 1 degree, and SN 1006 is an example. The diffuse Galactic $\gamma$-ray background is large at these energies due to the steep spectrum of the Galactic CRs that produce it, as pointed out by Drury et al. (1994). Therefore, we expect SNRs to be best observable at much higher $\gamma$-ray energies $\epsilon_{\gamma} \gtrsim 100 \mathrm{GeV}$, where the hard source spectra typically dominate over the background emission. Secondly, from a strictly empirical point of view, such a nuclear CR component that produces the $\pi^{0}$-decay bump need not extend far beyond the $\mathrm{GeV}$ region. For the existence, in a SNR, of a nuclear CR component approaching the energy region of the knee in the Galactic spectrum, the detection of a $67.5 \mathrm{MeV} \gamma$-ray feature is therefore neither a necessary nor a sufficient condition.

On the other hand, due to the high magnetic field strength which we deduce for this remnant, the $\gamma$-ray spectra produced by the electronic and hadronic CR components have rather similar shapes in the energy interval $0.1 \mathrm{GeV} \lesssim \epsilon_{\gamma} \lesssim 10 \mathrm{TeV}$ due to electron synchrotron losses. As a consequence the best observational possibility to discriminate between leptonic and hadronic contributions at high energies $\gg 1 \mathrm{GeV}$ is to measure the $\gamma$-ray spectrum at energies higher than $10 \mathrm{TeV}$, where we expect these two spectra to be essentially different. Therefore the clear detection of a substantial flux at energies $\epsilon_{\gamma} \gtrsim 10 \mathrm{TeV}$ would provide direct evidence for its hadronic origin.

\section{Inefficient proton acceleration model}

The set of parameters which was discussed until now is limited by the existing measurements. There is almost no freedom for a substantial change of any parameter value without a loss of agreement between theory and experiment. For example, the proton injection rate which determines the number of accelerated protons and the $\pi^{0}$-decay $\gamma$-ray emission, was taken to produce the shock modification which in turn is re- quired to produce a steep electron spectrum $N_{\mathrm{e}} \propto p^{-2.1}$ needed to fit the observed radio emission of SN 1006. For this injection rate $\eta=2 \times 10^{-4}$ a relatively high upstream magnetic field is unavoidably required in order to produce the observed synchrotron emission.

On the other hand one may dismiss the relevance of a deviation of the observed radio spectrum from the form $S_{v} \propto v^{-0.5}$, and of a likely thermal contribution to the soft X-ray flux around $10^{17} \mathrm{~Hz}$, and try to reproduce all the observed emissions by effects of electrons alone (e.g. Pohl 1996; Mastichiadis \& de Jager 1996; Yoshida \& Yanagita 1997; Aharonian \& Atoyan 1999). The number of injected and accelerated protons and all the effects which they can produce in SN 1006 is suggested to be negligibly small in this extreme case. For example, the proton contribution to the $\mathrm{TeV} \gamma$-ray emission should be at least an order of magnitude lower compared with the previous case. At such low proton numbers the nonlinear shock modification due to their backreaction is negligible, and shock acceleration takes place in the test particle limit.

In order to make clear how different the required set of relevant physical parameters compared to the case of efficient $\mathrm{CR}$ nucleon acceleration is, we performed a calculation which corresponds to inefficient $\mathrm{CR}$ acceleration. We use a proton injection rate $\eta=10^{-5}$ which yields an upper limit for the proton acceleration efficiency that is consistent with their low contribution to the $\gamma$-ray production. This low CR nucleon production efficiency also implies that the effect of magnetic field amplification due to CR streaming is small. Therefore we have used a typical upstream ISM magnetic field strength $B_{0}=4 \mu \mathrm{G}$. We note that such a low value was even assumed to be required in the compressed downstream region $-B_{\perp}=4 \mu \mathrm{G}$ - to fit the experimental data (Tanimori et al. 2001). All other ISM and SN parameters are the same as in the previous case of efficient CR acceleration. Since during the early evolutionary phase the global SNR dynamics is rather insensitive to the number of accelerated CRs, the observed SNR size and its expansion rate are equally well reproduced in this case.

In this test particle regime the electron spectrum, produced by a strong unmodified shock, can be analytically approximated by (Berezhko 1996)

$N_{\mathrm{e}} \propto p^{-\gamma} \exp \left[-\kappa(p) / \kappa\left(p_{\max }^{\mathrm{e}}\right)\right]$,

with $\gamma \approx 2$, where $p_{\max }^{\mathrm{e}}$ is the maximum CR momentum, which is determined by geometrical factors. Any essential deviation from $\gamma \approx 2$ would be inconsistent with diffusive shock acceleration which is believed to be the main process of energetic particle production in SNRs. For a Bohm-type diffusion coefficient, $\kappa \propto p$, the cutoff region of this spectrum has the same form $N_{\mathrm{e}} \propto \exp \left(-p / p_{\max }^{\mathrm{e}}\right)$ as it was suggested by Mastichiadis $\&$ de Jager (1996) and by Tanimori et al. (2001). At the same time, cf. Eq. (19), the spectrum of the electrons accelerated at a strong unmodified shock is essentially harder compared to what was used $(\gamma=2.2)$ to fit the radio, X-ray and $\gamma$-ray data (Tanimori et al. 2001). It is clear that the spectrum cf. Eq. (19), with $\gamma=2$ which has the same number of $\mathrm{GeV}$ electrons to produce the same radio flux, has an order of magnitude more electrons with $\epsilon_{\mathrm{e}}=10 \mathrm{TeV}$ compared with the case $\gamma=2.2$. Therefore, to fit the radio, X-ray, and $\gamma$-ray data with this harder 


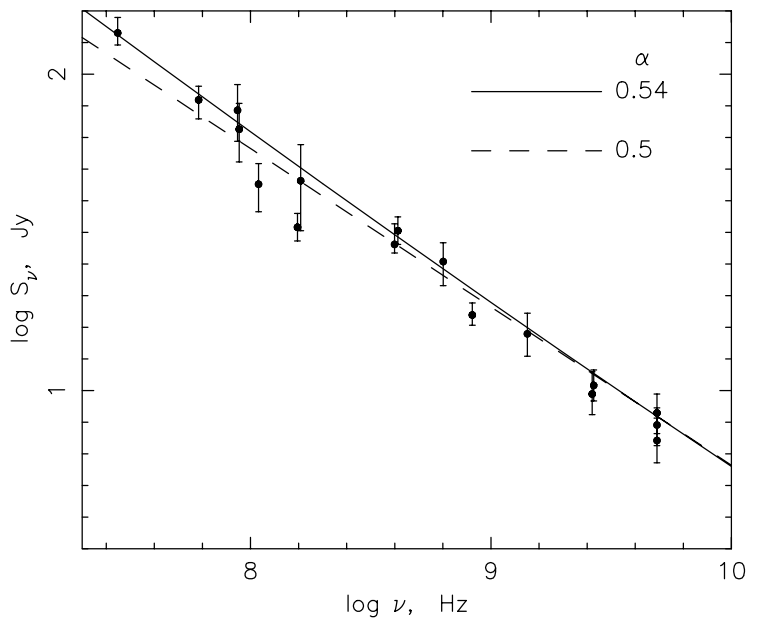

Fig. 6. Observed total radio flux of SN 1006 (Reynolds 1996) as a function of frequency with model spectra superimposed. Solid and dashed curves correspond in this figure to the high and low proton injection/acceleration efficiency, respectively.

electron spectrum, we need a large downstream magnetic field $B_{\perp} \approx 13 \mu \mathrm{G}$. Since even for $\eta=10^{-5}$ nucleon acceleration can produce a strong Alfvénic wave field, the upper limit for the downstream field strength $B_{\mathrm{d}}$ equals $16 \mu \mathrm{G}$. To this extent $B_{\perp} \approx 13 \mu \mathrm{G}$ can still be consistent with a $4 \mu \mathrm{G}$ ISM magnetic field as used by Tanimori et al. (2001), although these authors used $4 \mu \mathrm{G}$ also downstream. Since the energies $\epsilon_{\mathrm{e}} \gtrsim 10^{13} \mathrm{eV}$ of electrons, whose synchrotron emission in the given magnetic field corresponds to X-ray energies $\epsilon_{v}>5 \mathrm{keV}$, should be in the exponential cutoff region, the required value of the maximum $\mathrm{CR}$ momentum is $p_{\max }^{\mathrm{e}}=10^{4} m_{\mathrm{p}} c$. If we assume the same momentum dependence $\kappa \propto p$, this maximum CR momentum is consistent with the diffusion coefficient

$\kappa(p)=5.3 \kappa_{\mathrm{Bohm}}(p)$,

which exceeds the Bohm limit by a factor 5.3 for an upstream field of $4 \mu \mathrm{G}$.

The results for this inefficient proton injection/acceleration case are also presented in Figs. 2-7. One can see from Fig. 2 that, due to the lower magnetic field value, one needs about five times more accelerated electrons compared with the previous case to fit the radio and X-ray data (see Fig. 3).

In the inefficient case the $\gamma$-ray production is dominated by the electron contribution (Fig. 4). Since synchrotron losses are not important, the predicted IC spectrum is essentially harder for $\gamma$-ray energies between about $10^{9}$ and $10^{11} \mathrm{eV}$ than in the efficient proton acceleration model. The differential $\gamma$-ray CANGAROO spectrum appears in better agreement with the $\pi^{0}$-decay emission of the efficient model than with the inefficient model IC prediction (Fig. 5).

In Fig. 6 the calculated synchrotron fluxes are compared with the experimental data in the radio range. One can see that the steeper spectrum $S_{v} \propto v^{-0.54}$ which corresponds to the efficient $\mathrm{CR}$ acceleration case gives a better fit to the radio data than the spectrum $S_{v} \propto v^{-0.5}$ corresponding to the case of inefficient proton injection, even though the experimental accuracy does not very clearly distinguish between these to variants.

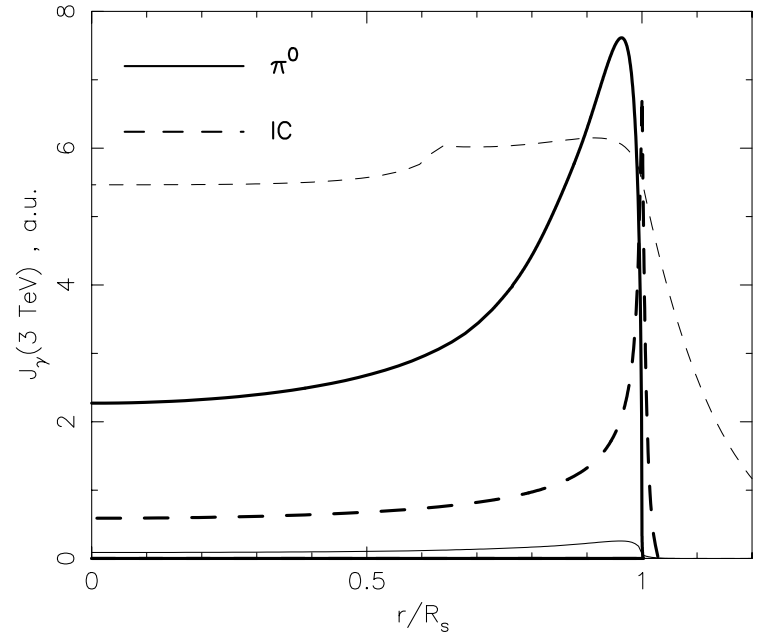

Fig. 7. Radial dependence of the $\gamma$-ray brightness for the $\gamma$-ray energy $\epsilon_{\gamma}=3 \mathrm{TeV}$. Thick and thin curves correspond to the high and low proton injection/acceleration efficiency, respectively.

Note that $\alpha=0.54$ is the average value of the power-law index within the frequency range shown in Fig. 6. In fact, due to the concave shape of the electron spectrum, the index slightly decreases with increasing frequency, with $\alpha=0.56$ and $\alpha=0.51$ at the lowest and largest frequency, respectively.

A similar situation is encountered in the X-ray range. An essentially steeper electron spectrum in the case of efficient CR accelerations much better fits the nonthermal X-ray data by lying below the total X-ray flux near the frequency $v=10^{17} \mathrm{~Hz}$ which corresponds to the photon energy $\epsilon_{v}=0.4 \mathrm{keV}$ (Fig. 3).

At given number of protons and magnetic field strength an electron to proton ratio $K_{\text {ep }}=0.04$ is required in the inefficient case, in order to reproduce the observed emission.

Nucleonic CRs absorb in this case only

$E_{\mathrm{c}}=0.008 E_{\mathrm{sn}}=2.4 \times 10^{49} \mathrm{erg}$,

which is more than an order of magnitude less than the canonically required $0.1 E_{\mathrm{sn}}$ for the Galactic CRs. The electron CR component contains $E_{\mathrm{c}}^{\mathrm{e}} \approx 10^{48} \mathrm{erg}$. This is a factor 7 less than what has been obtained by Dyer et al. (2001) corresponding to their lower downstream magnetic field.

It is important to note that the $\gamma$-ray brightness of the remnant

$J_{\gamma}\left(\epsilon_{\gamma}, r\right) \propto \int \mathrm{d} x q_{\gamma}\left(\epsilon_{\gamma}, r, x\right)$

has a very different dependence upon the distance from its center $r$ in the two considered cases. The integration of the $\gamma$-ray emissivity $q_{\gamma}$ (which is the number of photons emitted per unit volume per second in the range $\epsilon_{\gamma}$ to $\epsilon_{\gamma}+\mathrm{d} \epsilon_{\gamma}$ ) in the above expression is performed along the line of sight which intersects the visible remnant surface at the projected distance $r$ from its center. In the case of inefficient proton injection $\mathrm{TeV} \gamma$-rays are produced by the electrons with the highest energies of their spectrum $\epsilon_{\mathrm{e}} \gtrsim \epsilon_{\max }^{\mathrm{e}}$. These electrons almost uniformly occupy the entire volume between the shock surface $R_{\mathrm{S}}$ and the contact discontinuity $R_{\mathrm{p}}$ (which separates the ejecta and swept-up matter) with only a slight increase of their density toward the shock 
position. Depending on the turbulence level near the contact discontinuity they also partially fill the ejecta volume $r \lesssim R_{\mathrm{p}}$. Since the upstream distribution of these electrons is characterized by a diffusive length which is of the order of $0.1 R_{\mathrm{s}}$, the region $r>R_{\mathrm{S}}$ provides a noticeable contribution to the $\gamma$-ray brightness. Therefore the $\mathrm{TeV} \gamma$-ray brightness $J_{\gamma}(r)$ will have a peak value in the region $R_{\mathrm{p}}<r<R_{\mathrm{s}}$ if particle penetration through the contact discontinuity is strongly suppressed, and at the center of the remnant $r=0$ in the opposite case. A significantly different radial profile $J_{\gamma}(r)$ is expected in the case of efficient proton production. $\mathrm{TeV} \gamma$-rays are produced in this case by protons whose energy is considerably lower than the cutoff energy $\epsilon_{\max }$. Therefore they are concentrated within the thin region of thickness $\Delta r \approx R_{\mathrm{s}} /(3 \sigma)$ just behind the shock. The swept-up gas has a similar distribution. Since the $\pi^{0}$-decay $\gamma$-ray luminosity is proportional to the product of the CR density and the gas number density, the $\gamma$-ray brightness $J_{\gamma}(r)$ is expected to have a sharp peak close to the shock edge $r \approx R_{\mathrm{S}}$ with a considerable decrease at small distances $r \ll R_{\mathrm{s}}$. The morphological effect of renormalization is to limit this emission to two polar caps where the interstellar magnetic field is parallel to the shock normal.

The $\gamma$-ray brightness calculated for the two different cases is presented in Fig. 7 for the $\gamma$-ray energy $\epsilon_{\gamma}=3 \mathrm{TeV}$. One can see the essentially different radial dependence $J_{\gamma}(r)$ for the two cases. The $\gamma$-rays of hadronic origin are expected to be concentrated in the postshock region, whereas the IC $\gamma$-ray brightness, calculated for quite a large level of turbulence near the contact discontinuity (see Berezhko \& Völk 2000 for details), is almost uniformly distributed across the visible disk of the remnant. As it is seen from Fig. 7, the IC high energy emission of the inefficient case produces a noticeable halo around the shock - for SN1006 its size is about 0.1 degrees - that can in principle be used to discriminate between the $\gamma$-ray emissions of hadronic and leptonic origin.

The reported $\gamma$-ray flux was detected from the same outer part of SN 1006 which shows the radio-emission. As it was already pointed out (Aharonian 1999; Aharonian \& Atoyan 1999; Berezhko et al. 1999; Berezhko et al. 2001), this can be considered as an observational argument favoring a strong role for the nuclear CR component.

\section{Summary and conclusions}

The nonlinear kinetic model for CR acceleration in SNRs has been applied to SN 1006 in order to explain its observed properties. We have used stellar ejecta parameters $M_{\mathrm{ej}}=1.4 M_{\odot}$, $k=7$, distance $d=1.8 \mathrm{kpc}$, and ISM number density $N_{\mathrm{H}}=$ $0.3 \mathrm{~cm}^{-3}$ from X-ray and optical imaginary of SN 1006.

For these parameters an explosion energy $E_{\mathrm{sn}}=3 \times 10^{51} \mathrm{erg}$ is required to fit the observed size $R_{\mathrm{S}}$ and expansion speed $V_{\mathrm{S}}$ which are determined by the ratio $E_{\mathrm{sn}} / N_{\mathrm{H}}$.

The number of accelerated electrons required to fit the radio and X-ray emission of SN 1006, and correspondingly the role of accelerated protons in $\gamma$-ray production, depends essentially on the magnetic field value $B_{0}$.

It was demonstrated that for low magnetic field $B_{0}=4 \mu \mathrm{G}$ all the observed emissions can be dominated by the electron contribution. Protons are then assumed to be injected into the acceleration much less efficiently than electrons. For this test particle case the lowest permitted value of the electron to proton ratio is $K_{\mathrm{ep}}=0.04$. It exceeds the canonical value $0.01 \mathrm{ob}-$ served in situ in the neighborhood of the Solar System for the Galactic CRs. The maximum energy of accelerated CRs and their total energy content in this case are only $\epsilon_{\max } \sim 10^{13} \mathrm{eV}$ and $E_{\mathrm{c}}<3 \times 10^{49} \mathrm{erg}$ respectively. These numbers are too low for such SNRs to be considered as the main sources of the nucleonic Galactic CRs.

If CRs in SN 1006 are produced due to the diffusive shock acceleration process, then even in the case of inefficient proton injection quite a large but plausible downstream magnetic field $B_{\mathrm{d}} \approx 13 \mu \mathrm{G}$ is required to fit the data. It is several times larger than assumed in a simple estimate by Tanimori et al. (2001), because the shock produces in this case an electron spectrum $N_{\mathrm{e}} \propto \epsilon_{\mathrm{e}}^{-2}$ which is significantly harder than the spectrum assumed in that estimate.

The existing SNR data are better approximated if a significantly larger upstream magnetic field value $B_{0}=20 \mu \mathrm{G}$ and a physically much more plausible, efficient nucleon injection rate are assumed. Such an ion injection rate is estimated from injection theory, and consistent with the observed radio spectral index. The required magnetic field strength, that is significantly higher than the rms value $5 \mu \mathrm{G}$ in the ISM, might be the result of non-linear amplification near the SN shock by the CR acceleration process itself.

The results for this case of efficient proton acceleration are then as follows:

We find that after adjustment of the predictions of the nonlinear spherically-symmetric model by a renormalization of the number of accelerated nuclear CRs to take account of the large area of quasiperpendicular shock regions in a SNR, good consistency with all observational data can be achieved, including the reported $\mathrm{TeV} \gamma$-ray flux. The $\pi^{0}$-decay $\gamma$-ray flux produced by the nuclear CR component exceeds the flux of IC $\gamma$-rays generated by the electronic $\mathrm{CR}$ component at all energies above about $100 \mathrm{MeV}$. The theory did not make use of any knowledge to be derived from $\gamma$-ray measurements. Therefore the reported $\mathrm{TeV}$ flux from SN 1006 supports the idea that the nuclear CR component is indeed produced in SNRs. The $\pi^{0}$-decay $\gamma$-ray flux comes from two polar caps of the remnant.

The maximum energy of accelerated protons $\epsilon_{\max }=3 \times$ $10^{14} \mathrm{eV}$ and their total energy content $E_{\mathrm{c}} \approx 3 \times 10^{50} \mathrm{erg}$, reproduced in this case, are consistent with the requirements for the Galactic CR sources. The electron to proton ratio of $K_{\text {ep }}=1.5 \times 10^{-3}$, on the other hand, is lower than the canonical value 0.01 .

Comparing the case of efficient proton acceleration with the inefficient proton acceleration case, we see that the expected $\pi^{0}$-decay $\gamma$-ray flux $F_{\gamma}^{\pi} \propto \epsilon_{\gamma}^{-1}$ extends up to almost $100 \mathrm{TeV}$, whereas the IC $\gamma$-ray flux reaches less than about $10 \mathrm{TeV}$. Therefore the detection of $\gamma$-ray emission above $10 \mathrm{TeV}$ would imply evidence for a hadronic origin.

We therefore conclude that the analysis of SN 1006 on the basis of overall SNR dynamics and nonlinear diffusive shock acceleration theory results in a picture where the nuclear component is strongly accelerated, consistent with all data for this SNR. 
The ratio of accelerated electrons to protons comes out lower by a factor of about 6 than the canonical ratio of 0.01 , generally deduced from observations of the Galactic CRs. If SN 1006 is typical for the nuclear Galactic CR source population, then other sources like young Pulsars or Pulsar Nebulae must also significantly contribute to the Galactic CR electron population. The Crab Nebula is a point in case. Since the majority of Galactic Supernovae is not of the type Ia to which SN 1006 belongs, but rather occurs as a consequence of core collapse events, there is the alternative possibility that core collapse Supernovae produce a higher electron to proton ratio.

Phenomenological studies of the $\gamma$-ray emission from SN 1006 on the basis of the observed synchrotron emission have preferred a dominance of IC emission from electrons in the $\gamma$-ray part of the emission spectrum. This scenario can not explain the apparent bipolar morphology inferred from the existing $\gamma$-ray measurements and yields a much less convincing approximation to the radio and X-ray synchrotron spectrum. In addition, as we have shown, the IC emission should reach at best about $10 \mathrm{TeV}$. Apart from future detailed determinations of the $\gamma$-ray morphology one therefore needs to precisely measure the $\gamma$-ray flux at energies $\epsilon_{\gamma}$ between $100 \mathrm{GeV}$ and $100 \mathrm{TeV}$, and preferably even from $10 \mathrm{MeV}$ upwards. The detailed spectra and in particular the existence of $\gamma$-rays with very high energies above $10 \mathrm{TeV}$ should allow a confirmation, or a rejection, of CR nucleon production in SN 1006 with an acceleration efficiency that is consistent with the requirements on the Galactic CR energy budget.

Acknowledgements. This work has been supported in part by the Russian Foundation for Basic Research (grants 00-02-17728, 99-02-16325). EGB and LTK acknowledge the hospitality of the MaxPlanck-Institut für Kernphysik, where part of this work was carried out.

\section{References}

Aharonian, F. A. 1999, Astropart. Phys., 11, 225

Aharonian, F. A., \& Atoyan, A. M. 1999, A\&A, 351, 33

Aharonian, F. A., Atoyan, A. M., \& Völk, H. J. 1995, A\&A, 294, L41

Allen, W. H, Bond, I. A., Budding, E., et al. 1995, in Proc. 24th ICRC, Paris, 2, 447

Allen, G. E., Gotthelf, E. V., \& Petre, R. 1999, in 26th ICRC, Salt Lake City, 3, 480

Allen, G. E., Petre, R., \& Gotthelf, E. V. 2001, ApJ, 558, 739

Baring, M. G., Ellison, D. C., Reynolds, S. P., et al. 1999, ApJ, 513, 311
Bennet, L., \& Ellison, D. C. 1995, JGR, 100, 3439

Berezhko, E. G. 1996, Astropart. Phys., 5, 367

Berezhko, E. G., Elshin, V. K., \& Ksenofontov, L. T. 1996, JETP, 82, 1

Berezhko, E. G., \& Völk, H. J. 1997, Astropart. Phys., 7, 183

Berezhko, E. G., Ksenofontov, L. T., \& Petukhov, S. I. 1999, in Proc. 26th ICRC, Salt Lake City, vol. 3, 431

Berezhko, E. G., \& Ksenofontov, L. T. 1999, JETPh, 89, 391

Berezhko, E. G., \& Völk, H. J. 2000, A\&A, 357, 283

Berezhko, E. G., Ksenofontov, L. T., \& Völk, H. J. 2001, in 27th ICRC, Hamburg, 5, 2489

Berezinskii, V. S., Bulanov, S. A., Dogiel, V. A., et al. 1990, Astrophysics of cosmic rays (North-Holland: Publ. Comp.)

Blumenthal, G. R., \& Gould, R. J. 1970, Rev. Mod. Phys., 42, 236

Chevalier, R. A. 1982, ApJ, 258, 790

Dermer, C. D. 1986, A\&A, 157, 223

Drury, L. O'C., Aharonian, F. A., \& Völk, H. J. 1994, A\&A, 287, 959

Dyer, K. K., Reynolds, S. P., Borkowski, K. J., et al. 2001, ApJ, 551, 439

Ellison, D. C., Baring, M. G., \& Jones, F. C. 1995, ApJ, 453, 873

Jones, E. M., Smith, B. W., \& Straka, W. C. 1981, ApJ, 249, 185

Hamilton, A. J. S., Sarazin, C. L., \& Szymkowiak, A. E. 1986, ApJ, 300, 698

Koyama, K., Petre, R., Gotthelf, E. V., et al. 1995, Nature, 378, 255

Lucek, S. G., \& Bell, A. R. 2000, MNRAS, 314, 65

Malkov, M. A. 1998, Phys. Rev. E, 58, 4911

Malkov, M. A., \& Völk, H. J. 1995, A\&A, 300, 605

Malkov, M. A., \& Völk, H. J. 1996, Adv. Space Res., 21, No. 4, 551

Malkov, M. A., \& Drury, L. O'C. 2001, Rep. Prog. Phys., 64, 429

Malkov, M. A., Diamond, P. H., \& Jones, T. W. 2001, ApJ, 571, 856

Mastichiadis, A. 1996, A\&A, 305, L53

Mastichiadis, A., \& de Jager, O. C. 1996, A\&A, 311, L5

Moffett, D. A., Goss, W. M., \& Reynolds, S. P. 1993, AJ, 106, 1566

Naito, T., \& Takahara, F. 1994, J. Phys. G: Nucl. Part. Phys., 20, 477

Naito, T., Yoshida, T., Mori, M., \& Tanimori, T. 1999, Astronomische Nachrichten, 320, 205

Pohl, M. 1996, A\&A, 307, L57

Pohl, M., \& Esposito, J. 1998, ApJ, 507, 327

Reynolds, S. P. 1996, ApJ, 459, L13

Reynolds, S. P., \& Gilmore, D. M. 1993, AJ, 92, 1138

Scholer, M., Trattner, K. J., \& Kucharek, H. 1992, ApJ, 395, 675

Stecker, F. W. 1971, Cosmic Gamma Rays, NASA SP-249, chapter $5 \mathrm{ff}$.

Tanimori, T., Hayami, Y., Kamei, S., et al. 1998, ApJ, 497, L25

Tanimori, T., Naito, T., Yoshida, T., et al. 2001, in 27th ICRC, Hamburg, vol. 6, 2465

Trattner, K. J., \& Scholer, M. 1994, JGR, 99, 6637

Völk, H. J., et al. 2002, in preparation

Winkler, P. F., \& Long, K. S. 1997, ApJ, 491, 829

Yoshida, T., \& Yanagita, S. 1997, in 2nd INTEGRAL Workshop Transparent Universe (ESA SP-382), 85 